\title{
Aerosol dynamics modeling of silicon nanoparticle formation during silane pyrolysis: a comparison of three solution methods
}

\author{
Suddha S. Talukdar, Mark T. Swihart* \\ Department of Chemical and Biological Engineering, University at Buffalo (SUNY), \\ 506 Furnas Hall, Amherst Campus, Buffalo, NY 14260-4200, USA
}

Received 12 September 2003; received in revised form 5 February 2004; accepted 6 February 2004

\begin{abstract}
A numerical model has been developed to predict gas-phase nucleation, growth, and coagulation of silicon nanoparticles formed during thermal decomposition of silane. A detailed chemical kinetic model of particle nucleation was coupled to an aerosol dynamics model that includes particle growth by surface reactions, coagulation with instantaneous coalescence, and convective transport. Solution of the aerosol general dynamic equation was handled by three approaches: (1) the efficient and reasonably accurate method of moments; (2) the quadrature method of moments, which requires no prior assumption of the shape of the particle size distribution; and (3) a computationally more expensive sectional method (SM). The SM includes a conceptually simple and computationally efficient algorithm for treating coagulation that is found to be superior to widely used methods from the literature. All three approaches gave similar results for the evolution of the first few moments of the particle size distribution. The SM was able to capture the bimodal distribution that appears briefly at short residence times due to simultaneous nucleation and coagulation. At longer residence time, only coagulation remains important and both the sectional and moment methods give very similar results as they approach the self-preserving size distribution.
\end{abstract}

(c) 2004 Elsevier Ltd. All rights reserved.

Keywords: Silicon; Nanoparticles; Method of moments; Sectional method; Chemical nucleation; Aerosol dynamics; Nanoparticle formation; Silane pyrolysis

\section{Introduction}

A frequent by-product of silicon chemical vapor deposition (CVD) is the formation of powder from gas-phase chemical reactions that lead to homogeneous nucleation. In most cases, gas-phase

\footnotetext{
* Corresponding author. Tel.: +1-716-645-2911X2205; fax: +1-716-645-3822.

E-mail address: swihart@eng.buffalo.edu (M.T. Swihart).
} 
nucleation is viewed as an undesirable phenomenon that limits film growth rate and causes contamination (Girshick, Swihart, Suh, Mahajan, \& Nijhawan, 2000; Kremer, Davis, Moore, \& Ehrman, 2003). Particulate contamination is a leading cause of yield loss in semiconductor processing. As feature sizes in microelectronic circuits decrease, the critical particle size that leads to device failure decreases proportionately. In many cases, the particles are formed by homogenous nucleation via chemical reaction. Fundamental understanding of the chemical nucleation process, as well as subsequent particle growth, coagulation and transport, is necessary if we are to effectively control particle formation during semiconductor processing. Intentional silicon nanoparticle synthesis is also of great interest, due to the size-dependent, efficient photoluminescence of these silicon nanoparticles that is not present in bulk silicon. Our group is synthesizing silicon nanoparticles in a laser-driven aerosol reactor ( $\mathrm{Li}, \mathrm{He}$, Talukdar, \& Swihart, 2003). It is important to understand the physico-chemical fundamentals of silicon particle formation in our reactor in order to control particle size and morphology.

Various mechanisms for the nucleation of silicon particles from silane have been proposed (Frenklach, Ting, Wang, \& Rabinowitz, 1996; Yuuki, Matsui, \& Tachibana, 1987). A detailed chemical clustering model was previously developed for chemical nucleation of silicon hydrides containing up to ten silicon atoms (Swihart \& Girshick, 1999), and that model was used here. The clustering model includes detailed information regarding the thermodynamic properties and reactivities of silicon hydride clusters. The mechanism also includes cyclic and polycyclic clusters, which are important for explaining the formation of 'stable' nuclei in the gas phase. The chemical nucleation model has to be coupled with the aerosol general dynamic equation (GDE) to predict the evolution of the particle size distribution. In Girshick et al. (2000) and Nijhawan et al. (2003), a moment method assuming a lognormal distribution was used to solve the GDE in conjunction with this detailed nucleation model for simple one-dimensional geometries. Here we compare that approach to methods that provide greater detail without making a priori assumptions about the shape of the size distribution.

The aerosol GDE represents a population balance of particles undergoing internal processes of nucleation, growth and coagulation along with the external transport processes of diffusion, convection and thermophoresis. The GDE is a non-linear, partial integro-differential equation for which there is usually no analytical solution. Hence, various numerical techniques have been used to obtain approximate solutions to the problem. When particle formation occurs in a chemically reacting flow, the GDE must be coupled to the appropriate mass, momentum, energy, and chemical species balances. With increased computational power, it is increasingly possible to simultaneously capture details of the fluid flow and transport, the evolution of the aerosol size distribution and complex chemical kinetics. However, to obtain a solution at reasonable computational cost, researchers constantly have to make trade-offs in the level of detail included in each area. Our goal is to provide a framework for simultaneously incorporating detailed chemical kinetics in fluid flow and transport models and coupling these with an accurate, yet computationally tractable, aerosol dynamics model (Talukdar, $\mathrm{Ng}, \&$ Swihart, 2003). Similar efforts to model detailed aerosol processes of simultaneous nucleation, growth and coagulation have been developed for other systems (Prakash, Bapat, \& Zachariah, 2003; Moody \& Collins, 2003) using nodal or multi-modal approaches. However, a simplified sectional model for silicon particle formation along with detailed chemical kinetics has not been previously presented.

Moment models, typically solving for the first three moments of the particle volume distribution, are simple to implement. With relatively small computational effort, these can give representative properties of the aerosol size distribution such as total particle concentration, mean size and 
polydispersity. However, conventional moment methods have the disadvantage that the shape of the particle size distribution must be specified in advance to obtain closure of the equations (Pratsinis \& Kim, 1989). This could be a limitation if size distributions at early stages of particle formation are to be studied, where bimodal distributions are often observed (Landgrebe \& Pratsinis, 1990; Park, Lee, \& Choi, 1999; Xiong \& Pratsinis, 1991). To avoid specifying the shape of the distribution a priori, the quadrature method of moments (QMOM) developed by McGraw (1997) approximates the integral moments of the size distribution by an $n$-point Gaussian quadrature (Gordon, 1968; Hulburt \& Katz, 1964). The QMOM does not define or produce an explicit size distribution, but the moments could, in principle, be used with an assumed functional form to obtain a size distribution (Barrett \& Webb, 1998; McGraw, Nemesure, \& Schwartz, 1998; Diemer \& Olson, 2002). For comparison to experiment or other computational methods, having only a list of higher moments and not a size distribution or an easy means of obtaining quantities like the geometric standard deviation is a significant disadvantage of the QMOM method, as discussed further below.

Sectional methods (SM), based on dividing the continuous particle size distribution into discrete sections and dealing with one integral property at a time have been extensively used for more than 20 years (Gelbard \& Seinfeld, 1980; Hounslow, Ryall, \& Marshall, 1988; Kumar \& Ramkrishna, 1996a; Landgrebe \& Pratsinis, 1990; Xiong \& Pratsinis, 1991). Coagulation is generally the most computationally expensive part of aerosol dynamics calculations using SM, due to the presence of complex kernels and the fact that it involves double summations over the particle size bins to account for coagulation of each size with all other sizes. This becomes demanding if aerosol dynamics are to be coupled with multidimensional computational fluid dynamics models (Johannessen, Pratsinis, \& Livbjerg, 2000; Muhlenweg, Gutsch, Schild, \& Pratsinis, 2002; Schild, Gutsch, Muhlenweg, \& Pratsinis, 1999). It may be important to use a SM to capture the early stages of particle formation. We have thus implemented a SM (referred to below as STMS code) with a simple treatment of coagulation based on conserving the mean volume of two particles from different bins colliding to form a third particle in some other bin. This method is similar to the general approach used by Kumar and Ramkrishna (1996a) for population balances involving coagulation and breakage. In this case, we couple this treatment of coagulation, conserving particle number and volume, with particle nucleation and growth by chemical reaction.

Surface growth generally introduces numerical diffusion for fixed SM as noted in Wu and Flagan (1988) and Kim and Seinfeld (1990). Moving SM (Kim \& Seinfeld, 1990; Kumar \& Ramkrishna, 1997; Spicer, Chaoul, Tsantilis, \& Pandis, 2002) can eliminate numerical diffusion by surface growth and have been used in the past quite successfully. However, these are not easily integrated into multi-dimensional simulations where one wants to use the same set of size bins at every spatial point in the simulation. In our effort to develop this computationally feasible framework for coupled aerosol dynamics and detailed chemical kinetics, we have used a simple 'two-point' method of discretization for surface growth, similar to that used in Hounslow et al. (1988). Numerical dispersion could be an important issue, especially for evolution of initially narrow size distributions when there is substantial surface growth. However, for the simultaneous nucleation, coagulation, and growth processes modeled here, where surface growth contributes but is never the dominant or only contributor to evolution of the size distribution, the simple two-point method used here provides reasonable accuracy at low computational cost. We did observe slight broadening of the size distributions with decreasing number of sections, vide infra, but the changes were small, suggesting that numerical diffusion is not important in this case. 
As implemented here, our method is both conceptually simple and computationally efficient relative to SM that have been widely used in aerosol dynamics modeling. This simplicity and efficiency is highly desirable for problems like the one at hand, where there are complexities both in the chemical reaction kinetics and the aerosol dynamics and therefore the aerosol dynamics cannot be the sole focus of the overall solution procedure.

\section{Theory}

The aerosol GDE for simultaneous nucleation, growth and coagulation can, in principle, be solved for given initial and boundary conditions to give the particle size distribution function. The continuous GDE represents a population balance of the particles in the volume interval from $v$ to $v+\mathrm{d} v$ and is given in its general form by the following equation:

$$
\begin{aligned}
\frac{\partial n(v)}{\partial t} & +\nabla \cdot V n(v)-\nabla \cdot D(v) \nabla n(v)+\nabla \cdot V_{\mathrm{th}} n(v) \\
= & \frac{1}{2} \int_{0}^{v} \beta(\bar{v}, v-\bar{v}) n(\bar{v}) n(v-\bar{v}) \mathrm{d} \bar{v}-n(v) \int_{0}^{\infty} \beta(\bar{v}, v) n(\bar{v}) \mathrm{d} \bar{v} \\
& -\frac{\partial}{\mathrm{d} v}\left(n(v) \frac{\mathrm{d} v}{\mathrm{~d} t}\right)+I\left(v^{*}\right) \delta\left(v-v^{*}\right) .
\end{aligned}
$$

Here $V$ is the convective velocity, $D(v)$ is the Brownian diffusion coefficient for a particle of size $v, V_{\text {th }}$ is the particle thermophoretic velocity, $\beta$ is the particle collision frequency function and $I$ is the nucleation rate. The first term on the left-hand side (LHS) describes the rate of change of particle concentration while the next three terms represent loss or gain of particles due to convection, diffusion and thermophoresis, respectively. These are collectively referred to as the external processes as they involve movement of particles across the control volume boundary. For the steady-state, isothermal, one-dimensional plug flow reactor that was used to develop the aerosol model described here, the transient term, diffusion term and thermophoresis term are omitted. The first two terms on the right-hand side (RHS) of Eq. (1) represent gain and loss of particles due to Brownian coagulation (Friedlander, 2000). The collision frequency $\beta$ is based on the harmonic mean approximation (Lee \& Chen, 1984; Pratsinis, 1988) to interpolate between the free molecular and continuum regimes, and is described in detail in Appendix A.1. The third term on the RHS of Eq. (1) is related to particle growth, which is treated as a chemical vapor deposition (CVD) process where the gas-phase species react on the particle surface by reactions like those in the silicon film growth mechanisms presented by Ho, Coltrin, and Breiland (1994). The particle growth rate by surface reactions was calculated self-consistently using species concentrations determined from the gas-phase chemical kinetic model. The gas-phase species equations were also fully coupled to the particle growth by properly accounting for species depletion as a result of surface reactions (Girshick et al., 2000). Chemical nucleation of particles is based on a chemical clustering model (Swihart \& Girshick, 1999), in which the basic mechanism includes reversible chemical reactions between silicon/hydrogen molecules containing up to ten silicon atoms. The nucleation rate is taken to be the rate at which these chemical reactions irreversibly produce molecules containing more than ten silicon atoms. This critical nucleus size of ten silicon atoms is presently just a coarse estimate, but in the future can hopefully be obtained from 
kinetic Monte Carlo simulations of cluster formation, as presented in Li and Swihart (2001), or from the automated reaction mechanism generation approach (Broadbelt, Stark, \& Klein, 1994; Broadbelt, Stark, \& Klein, 1996) being applied to this system by Wong, Li, Swihart, and Broadbelt (2003). The observations of Nijhawan et al. (2003) suggest that the present nucleation model overpredicted the nucleation rate for the conditions that they studied.

\subsection{Method of moments (MOM)}

An efficient and reasonably accurate means of approximately solving the GDE is the MOM, which has been extensively used due to its relative ease of implementation and low computational cost (Lee, Chen, \& Gieseke, 1984; Nijhawan et al., 2003; Pratsinis \& Kim, 1989). The $k$ th moment of the particle size distribution function $n(v)$ is given by

$$
M_{k}=\int_{0}^{\infty} v^{k} n(v) \mathrm{d} v
$$

where $n(v)$ is the particle size distribution in volume. The dynamic behavior of a lognormal distribution can be described from the first three moments of the particle size distribution in volume (Pratsinis, 1988). The zeroth moment $\left(M_{0}\right)$ represents the total particle concentration, the first moment $\left(M_{1}\right)$ gives the particle volume fraction (volume of particles per unit volume of gas) and the second moment $\left(M_{2}\right)$ is related to the light scattering intensity from particles. Using MOM, the GDE can be reduced to a set of three moment equations (Frenklach \& Harris, 1987; Mahajan, 1999; Pratsinis \& Kim, 1989). A major drawback of this method is that it requires that all terms in the moment equations be expressed as functions of the moments themselves. This closure of the moment equations is usually achieved by assuming the shape of the size distribution to be lognormal. Many experimental results suggest that aerosol size distributions are generally lognormal and the lognormal distribution has three parameters that are readily related to the first three moments of the size distribution (Pratsinis, 1988).

\subsection{Quadrature method of moments}

The QMOM avoids using an assumed shape for the size distribution by approximating the integral moments by an $n$-point Gaussian quadrature (Lanczos, 1988). This solves the problem of obtaining closure of the moment equations, so the coagulation, growth, diffusion and thermophoretic terms can be expressed in their original forms. The moments of the size distribution can be expressed as functions of abscissas and weights of the Gaussian quadrature:

$$
M_{k}=\int_{0}^{\infty} r^{k} n(r) \mathrm{d} r \approx \sum_{i=1}^{N q} f\left(r_{i}\right) \text { Weights }_{i} .
$$

The abscissas and weights are calculated from the moments through a product-difference (PD) algorithm from the lower-order moments (Marchisio, Vigil, \& Fox, 2003; Gordon, 1968). This method is an extension of the Hulburt and Katz (1964) procedure applied to aerosol dynamics problems 
by McGraw (1997). For a three-point quadrature approximation, six radial (not volume) moments $\left(M_{0}-M_{5}\right)$ are required and were used in our calculations. The QMOM does not define or produce an explicit size distribution and hence was used only for calculating the integral properties, but the six moments could be used with an assumed functional form for a size distribution with six degrees of freedom to produce one a posteriori (McGraw et al., 1998; Diemer \& Olson, 2002).

\subsection{Sectional method}

The SM approximates the continuous size distribution by a finite number of sections or bins within which one numerically conserved aerosol property is held constant (Gelbard, Tambour, \& Seinfeld, 1980; Hounslow et al., 1988; Kostoglou \& Karabelas, 1994; Kumar \& Ramkrishna, 1996a; Landgrebe \& Pratsinis, 1990). This is computationally more expensive than the two moment methods described above, but it is able to capture the details of early stages of particle formation where the assumption of a lognormal size distribution (for MOM) often breaks down. Using a finite element type formulation, the particle size distribution can be approximated as

$$
n(v) \approx \sum_{i=1}^{i_{\max }} n_{i} \phi_{i},
$$

where the $\phi_{i}$ are basis functions that are equal to one within bin $i$ (for $v_{i-1}<v<v_{i}$ ) and zero elsewhere; the $n_{i}$ are the coefficients of the discretized particle size distribution, which are constant within each bin; and $i_{\max }$ is the number of bins used (typically 100 in this work). Multiplying the GDE by $\phi_{i}$ and integrating over all $v$ from 0 to $\infty$ gives an equation for the number of particles in the size range $v_{i-1}<v<v_{i}$, which converts the GDE (Eq. (1)) into a set of $i_{\max }$ equations for the number of particles in each size range.

$$
\frac{\mathrm{d}}{\mathrm{d} x}\left(V n_{i}\right)=\frac{\text { Coagulation }_{\text {gain }, i}-\text { Coagulation }_{\text {loss }, i}}{v_{i}-v_{i-1}}+\frac{n_{i-1} \text { Growth }_{i-1}-n_{i} \text { Growth }_{i}}{v_{i}-v_{i-1}}+\frac{I_{\text {nucleation }, i}}{v_{i}-v_{i-1}} .
$$

The particle number concentration in each bin changes due to contributions from coagulation, nucleation, and growth. In treating coagulation, many researchers, including Landgrebe and Pratsinis (1990) and Gelbard et al. (1980) have used size-dependent inter-bin and intra-bin coagulation coefficients that lead to complicated integrals for these terms. We instead use a relatively simple treatment of coagulation that simplifies the calculation and decreases computational cost. This is similar to the approach adopted by Kumar and Ramkrishna (1996a), Hounslow et al. (1988) and Lister, Smit, and Hounslow (1995), who used a constant collision kernel within each bin to simplify the calculations. Our approach starts from the point of view of the coagulating particles, first computing the rate at which particles from each bin collide with those from another bin of the same or larger size. For the collisions between particles from bin $j$ and bin $k$, we first find the total coagulation rate between such particles:

$$
B_{\text {coagulation }(j, k)}=n_{j} n_{k} \beta\left(v_{\text {mean }, j}, v_{\text {mean }, k}\right)\left(v_{j}-v_{j-1}\right)\left(v_{k}-v_{k-1}\right) .
$$

Here $\beta\left(v_{\text {mean }, j}, v_{\text {mean, } k}\right)$ is constant for a particular $(j, k)$ pair, which is a reasonable assumption to make when using a large number of narrowly-spaced bins. $\beta\left(v_{\text {mean }, j}, v_{\text {mean }, k}\right)$ is the harmonic 

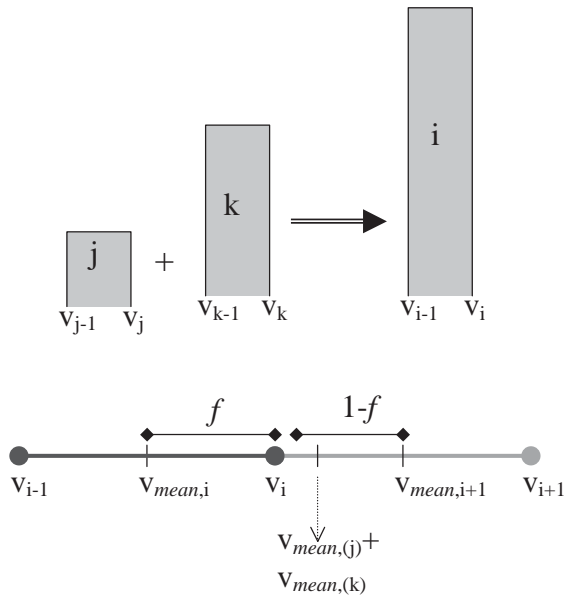

Fig. 1. Collision of two particles from a $(j, k)$ pair of bins is distributed among bin $i$ and bin $i+1$ such that particle number and volume are conserved.

mean average of the continuum and free-molecular regime as employed by Pratsinis (1988) and Xiong and Pratsinis (1991), shown in more detail in Appendix A. The spacing factor $\left(f_{\mathrm{s}}\right)$ is defined as the ratio of the width of bin $i+1$ to the width of bin $i$. In terms of the total number of bins, $i_{\max }$, and the maximum $\left(V_{\max }\right)$ and minimum $\left(V_{\min }\right)$ particle volumes considered, the spacing factor is defined by

$$
f_{\mathrm{s}}=\exp \left(\frac{\ln \left(V_{\max } / V_{\min }\right)}{i_{\max }}\right),
$$

where $V_{\max }$ and $V_{\min }$ are the maximum and minimum volumes of the size bins and $i_{\max }$ is the maximum number of size bins used. When particles from bin $j$ and bin $k$ (with mean volumes of $v_{\text {mean }, j}=\left(v_{j-1}+v_{j}\right) / 2$ and $v_{\text {mean }, k}=\left(v_{k-1}+v_{k}\right) / 2$, respectively) collide, the resulting particle could fall in some third bin, $i$, with mean volume $v_{\text {mean }, i}=\left(v_{i-1}+v_{i}\right) / 2$ or in the next bin, $i+1$, with mean volume $v_{\text {mean }, i+1}=\left(v_{i}+v_{i+1}\right) / 2$, where $v_{\text {mean }, i}<\left(v_{\text {mean }, j}+v_{\text {mean }, k}\right)<v_{\text {mean }, i+1}$ (Fig. 1$)$. In the formulation used here, the fraction of particles $(f)$ resulting from $(j, k)$ collisions that falls in bin $i$ is obtained from Eq. (8), which ensures that the coagulation process conserves particle volume (or mass) as well as particle number:

$$
v_{\text {mean }, j}+v_{\text {mean }, k}=f * v_{\text {mean }, i}+(1-f) * v_{\text {mean }, i+1} .
$$

Thus, the changes in particle number in bins $i, i+1, j$, and $k$ as a result of $(j, k)$ collisions is given by the following equations:

$$
\begin{aligned}
& \text { Coagulation }_{\text {gain }, i}=f \cdot B_{\text {coagulation }(j, k)}, \\
& \text { Coagulation }_{\text {gain }, i+1}=(1-f) \cdot B_{\text {coagulation }(j, k)} \text {, } \\
& \text { Coagulation }_{\text {loss }, j}=\text { Coagulation }_{\text {loss }, k}=B_{\text {coagulation }(j, k)} \text {. }
\end{aligned}
$$


Doing this for all $(j, k)$ pairs with $j \leqslant k$ and summing the contributions to each bin gives the total net change in particle number in each bin due to coagulation. For the case $j=k, B_{\text {coagulation must be }}$ divided by two to avoid double counting of collisions. This approach of conserving particle volume by a simple balance is similar to that used by Kumar and Ramkrishna (1996a). In the present model, we do not make a special effort to conserve the higher moments of the distribution, by conserving $v^{2}$ instead of $v$, for example, as it is the number and volume distributions that are of most interest in this case.

The third term on the RHS of Eq. (5) represents growth due to chemical reactions on the surface of each particle. As reviewed by Kumar and Ramkrishna (1997), growth terms introduce additional complexity in the problem due to dispersion of numerical solutions obtained using finite-difference approximations. Discontinuities are introduced as a result of combined nucleation and growth processes, and can result in negative values of the size distribution. Here we have used a simple upwind difference scheme (a standard 'two-point' method) where we compute the particle flux into and out of bin $i$ based on the growth rate and particle number in bins $i-1$ and $i$, respectively. While higher-order finite difference methods may provide more accurate results, as suggested by Kumar and Ramkrishna (1997), it seems unlikely that the improvements offered by using higher-order differencing schemes will significantly affect the integral aerosol properties that are of interest to us. Similar to the approach of Hounslow et al. (1988), the contribution of particle growth to the number of particles in bin $i$ is given by the difference between the rate at which particles from the smaller bin $(i-1)$ grow into the size range of bin $i$ minus the rate at which particles from bin $i$ grow into the next larger size bin $(i+1)$ :

$$
\text { Growth }_{i}=n_{i-1} * G_{\text {effective, } i-1}-n_{i} * G_{\text {effective, } i},
$$

where $G_{\text {effective }}$ is the harmonic mean average of the growth terms in the free molecular and continuum regimes:

$$
\begin{aligned}
& G_{\mathrm{CR}}=G_{\text {linear }} S\left(D_{\mathrm{m}} / k r\right), \\
& G_{\mathrm{FM}}=G_{\text {linear }} S,
\end{aligned}
$$

where the linear growth rate $G_{\text {linear }}$ is obtained from the rate of production of the bulk phase silicon per unit surface area by surface chemical reactions and is shown in Appendix A.2. $S$ is the total surface area of particles in the bin being considered $\left(S=\int_{v_{i-1}}^{v}(36 \pi)^{1 / 3} v^{2 / 3} \mathrm{~d} v\right), D_{\mathrm{m}}$ is the molecular diffusion coefficient of a representative growth species, $k$ is a representative surface reaction rate constant for deposition and $r$ is the particle radius. Silane is the representative growth species used here, as it is the dominant contributor to surface growth. The group $D_{\mathrm{m}} / k r$, whose inverse is the dimensionless surface Damköhler number for particle growth, approximately accounts for diffusional limitations on the particle growth rate. In order to maintain conservation of mass between the gas-phase species and particles, the depletion rates of gas-phase species were scaled to ensure that the amount of silicon removed from the gas phase by surface reaction was identical to that added to the particles during the growth process. Negative size distributions were avoided by simply neglecting growth if the population, $n_{i}$, in a given bin was below a fixed value (usually 20 orders of magnitude lower than that of the bin with the largest population) and the net growth contribution to that bin would lead to a decrease in $n_{i}$. 
Nucleation, the last term in Eq. (5) is obtained from the detailed chemical kinetic model of cluster formation as described above. In the SM, the first size bin was selected to have the same mean volume as the critical nucleus produced by the kinetic model. The nucleation rate was taken to be the formation rate of silicon-hydrogen clusters containing more than ten silicon atoms, as was done previously with this same reaction mechanism (Girshick et al., 2000; Nijhawan et al., 2003).

\section{Case study}

The numerical solution of all three methods was carried out for an isothermal plug flow reactor at atmospheric pressure. The chemical reaction and particle nucleation model included 1098 gas-phase reactions and 104 surface reactions involving 133 gas-phase, 2 surface phase and 1 bulk species. The simulation conditions were a constant temperature of $1023 \mathrm{~K}$, pressure of 1 atm and inlet silane mole fraction of $1 \%$ in helium carrier gas. For sectional simulations the initial size distribution was taken to be uniform, with a negligibly small particle number to avoid numerical difficulties encountered when starting from distribution with zero particles in each bin. Simulations utilized the Chemkin group of codes (Coltrin, Kee, Rupley, \& Meeks, 1996; Kee, Rupley, Meeks, \& Miller, 1996) for reaction rates and transport properties. The resulting differential equations were solved using DASSL (Petzold, 1982). The emphasis in this paper is on using a reliable, yet computationally efficient, aerosol dynamics model coupled to a large reaction mechanism to understand particle formation. We are trying to develop a framework for using detailed chemical kinetics coupled to aerosol dynamics and fluid flow in general and not just for the silicon particles mentioned here. So, although here we present results for this particular case, we expect this to be representative of what to expect both for improved chemical kinetic and transport models of silicon particle formation, and for other systems characterized by coupled complex chemical kinetics and aerosol dynamics such as soot formation and many flame and spray pyrolysis aerosol synthesis methods.

\section{Results}

\subsection{Validation}

The MOM results obtained here were validated against those presented by Girshick et al. (2000). To validate our implementation of the SM we compared its results to analytical solutions and a widely used numerical technique. This validation of the SM was carried out for coagulation as well as growth, based on analytical solutions of Gelbard and Seinfeld (1978), implemented by Hounslow et al. (1988). Compared to methods using complex coagulation kernels (Gelbard et al., 1980; Landgrebe and Pratsinis, 1990), Hounslow's code is computationally efficient because it assumes constant collision frequency within each interval. This dramatically reduces the complex integration required within each interval. The numerical error introduced as a result of this assumption can be substantially reduced by using a larger number of bins (or smaller $f_{\mathrm{s}}$ ), as shown in more detail below. The analytical solution for the coagulation only case (based on the sum kernel) was in excellent agreement with that obtained using our numerical method (STMS code, using a $f_{\mathrm{s}}$ value of 2) and was identical to the results from Hounslow's numerical scheme (also using a $f_{\mathrm{s}}$ value 
Table 1

Comparing relative CPU time for STMS and Lister et al. (1995, Table 2)

\begin{tabular}{llc}
\hline$f_{\mathrm{s}}$ & STMS & Lister \\
\hline 2 & 1 & 1 \\
1.41 & 1.98 & 8 \\
1.26 & 2.98 & 21 \\
1.19 & 3.99 & 49.5 \\
1.15 & 4.96 & 95.4 \\
1.12 & 5.98 & 167.7 \\
\hline
\end{tabular}

Note: The CPU time shown above is for coagulation only, with a constant coagulation kernel, run until the total particle number has decreased to $2 \%$ of its initial value.

of 2). For the simultaneous coagulation (size independent kernel) and surface growth case, numerical dispersion was observed at longer times, as expected for the simple upwind two-point finite difference scheme used here to describe growth. However as mentioned earlier, for the simultaneous nucleation, growth and coagulation of silicon particles, this numerical diffusion arising due to growth did not significantly affect the overall properties and can be safely ignored. In order to decrease the error introduced as a result of the assumption of constant collision frequency in each bin, $f_{\mathrm{s}}$ was decreased from 2 to 1.15 (while increasing the number of bins from 30 to 150, thereby keeping the same total volume range). Increasing the number of bins results in more accurate solutions as noted by Landgrebe and Pratsinis (1990). The flexibility of changing the spacing factor and thereby reducing the numerical error is also present in Lister et al. (1995) using an adjustable geometric size discretization with $f_{\mathrm{s}}$ given by $2^{1 / q}$ and integer values of $q$. However, compared to our simple model and relative ease of changing the spacing factor, the equations developed by Lister et al. (1995) are substantially more complicated, resulting in increased computational time. This can be seen from Table 1, which compares the relative CPU times for the size-independent collision kernel as shown in Table 2 of Lister et al. (1995). Primarily the increased computational time in their paper is a result of additional summation terms that are included for increasing values of $q$ (Table 1, Lister et al., 1995). The increase in CPU time was reported to be proportional to $q^{3}$ for the method of Lister et al., whereas for our code (STMS) the CPU times scales almost linearly with $q$. The dramatic reduction in the relative computational time (without loss of accuracy) suggests that the adjustable spacing factor in our simplified sectional model has the potential to make significant improvements in the efficiency of SM using large numbers of bins. The present method also accommodates non-constant spacing factors without any change in the algorithm. The only limitation is that the width of each bin be greater than or equal to the width of the bins at smaller particle size. Thus, it is possible to concentrate bins by using a finer grid (smaller spacing factor) in a size range of interest, or to use a series of discrete sizes at the lower end of the size distribution (a discrete-SM) without any special treatment of the coupling between 'discrete' and 'sectional' bins. The concept of selective refining of the grid has been used by Kumar and Ramkrishna (1996a,b), to obtain more accurate solutions without increasing the number of bins. From Fig. 2 (for the size-dependent collision kernel), it can be seen that decreasing $f_{\mathrm{s}}$ from 2 to 1.15 (thereby increasing the number of bins from 30 to 150 ) increases the computational time by $265 \%$ (from 139 to $509 \mathrm{~s}$ ), and reduces the root mean square (rms) error relative to the analytical solution by $91 \%$. In this case, the rms error $(y)$ scales with 


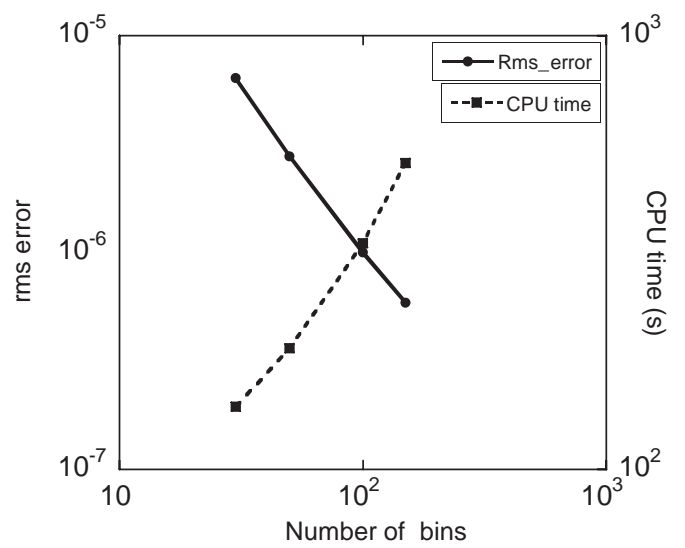

Fig. 2. Effect of changing number of bins from 30 to 150 (and hence $f_{\mathrm{s}}$ from 2 to 1.15 ) on the rms error relative to the analytical solution and on the CPU time are shown for the same case using the size-dependent (sum) collision kernel. The rms error $(y)$ scales with the number of bins $(x)$ as approximately the -1.5 power $\left(y=x^{-1.5}\right)$, while the CPU time increases as approximately the 0.8 power of the number of bins $\left(y=x^{0.8}\right)$.

the number of bins $(x)$ to approximately the -1.5 power $\left(y=x^{-1.5}\right)$, while the CPU time increase is proportional to the number of bins to approximately the 0.8 power $\left(y=x^{0.8}\right)$. Thus, increasing the number of bins will have a greater effect on lowering the rms error than on increasing the computational time. For coagulation alone, the SM was also tested to confirm that it approaches the self-preserving size distribution (Friedlander \& Wang, 1966; Friedlander, 2000), as has been done to validate other SM, such as that in Landgrebe and Pratsinis (1990). The geometric standard deviation of the self-preserving size distribution in the continuum and free molecular regimes was 1.451 and 1.469, respectively, which are similar to the values obtained by Vemury, Kusters, and Pratsinis (1994) for a volume-based sectional model using the same spacing factor.

The three methods that were used to solve the GDE are now compared with respect to some characteristic aerosol properties (particle concentration, volume fraction and particle diameter) with increasing residence time (in seconds) in the reactor for the case of silicon particle formation. Also plotted in Fig. 3 is the silane conversion, which is helpful to illustrate the interactions between chemical kinetics and aerosol dynamics. From Fig. 3, we see that the predictions for these three quantities of interest are in excellent agreement between the three methods. However looking at the inset in Fig. 3a, it can be seen that on a linear scale, the peak of the particle concentration is overpredicted by MOM by almost $15 \%$ as compared to the SM. The total particle concentration (that would be measured by standard particle counting instruments) can thus be measurably different between methods. However, this may not be significant from a modeling perspective for gas to particle conversion cases like this where we are unlikely to make predictions with this level of accuracy. The overall good agreement between the MOM and SM is however not always guaranteed, as considered in (Park et al., 1999) where the MOM and SM gave quite different results. From Fig. $3 \mathrm{a}$, we can see that the total particle concentration peaks around $2 \mathrm{~ms}$, which is the time scale for significant silane decomposition under these conditions (Girshick et al., 2000). Particle nucleation also peaks around this time, after which these particles become a sink for gas-phase species due to the large particle surface area on which surface reactions occur. The silane conversion verifies 

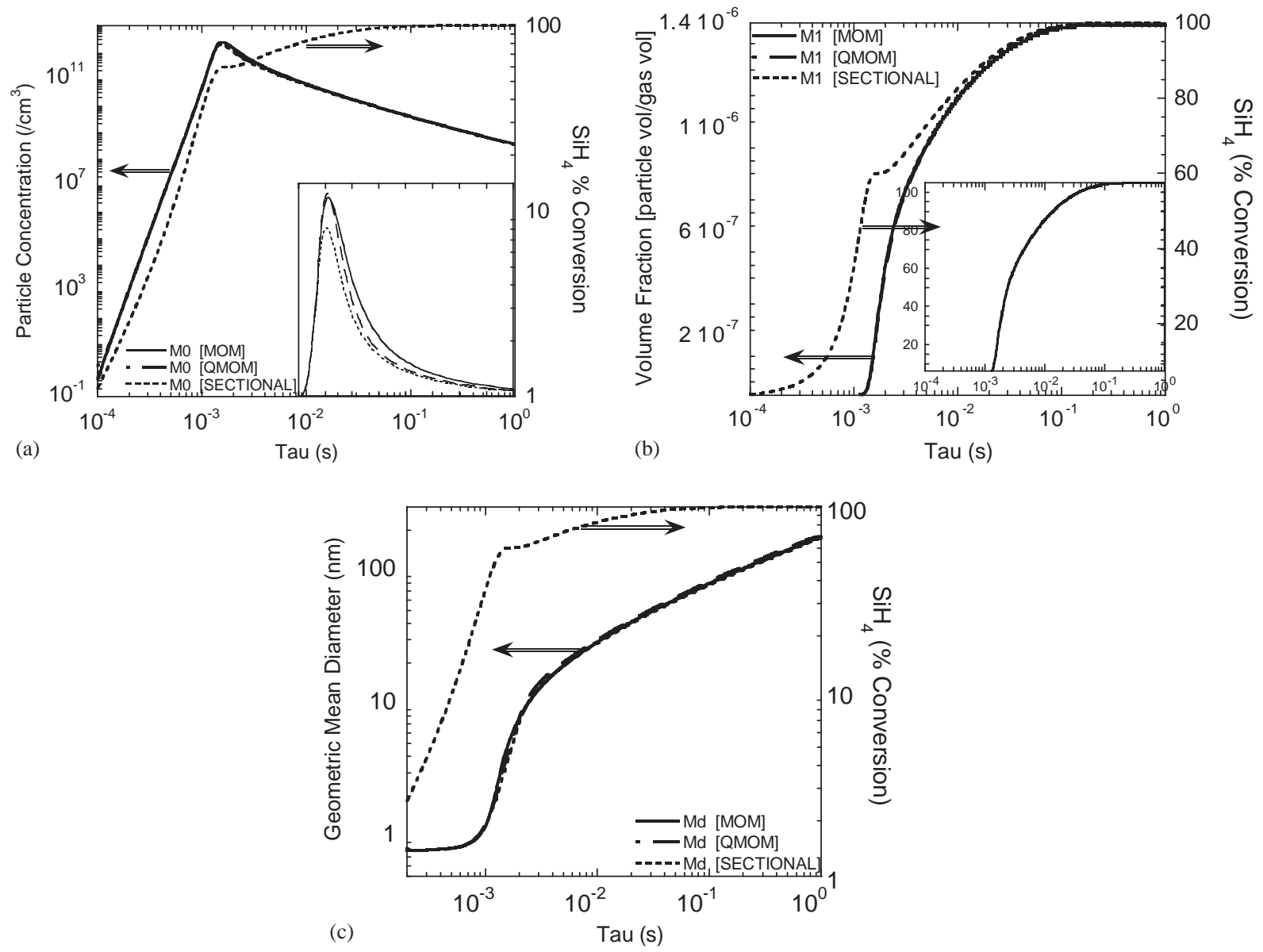

Fig. 3. Comparing MOM, QMOM and SM on (a) total particle concentration (on a logarithmic scale, and in the inset on a linear scale), (b) volume fraction and (c) mean particle diameter with increasing residence time in the reactor. Also included is the silane conversion, which helpful in identifying interactions between chemical reaction and aerosol dynamics.

that the sharp increase (with a slope equal to that of the particle concentration) is due to chemical nucleation and the silane decomposition rate peaks at the same time, around $2 \mathrm{~ms}$, after which the silane conversion slows down. Chemical reactions and, therefore, also some chemical nucleation, continue until a residence time of about $90 \mathrm{~ms}$, by which time all the silane gas has been converted to particles. Between 2 and $90 \mathrm{~ms}$, coagulation and surface growth are both active, and some nucleation is also still occurring. The prevailing mechanism after $90 \mathrm{~ms}$ is coagulation, which reduces the particle concentration with a corresponding increase in particle diameter (Fig. 3c). Particle volume fraction in Fig. $3 \mathrm{~b}$ shows a sharp increase around $2 \mathrm{~ms}$ and then a slower one up to a residence time of $90 \mathrm{~ms}$ when gas-phase species have been fully consumed by surface reactions (silane conversion is almost $100 \%)$. The surface growth mechanism is still active after the initial nucleation burst and adds significantly (about 64\%) to the total particle volume between 2 and $90 \mathrm{~ms}$, as can be more clearly seen in the normalized plot of volume fraction shown in the inset of Fig. 3b. It is worth 

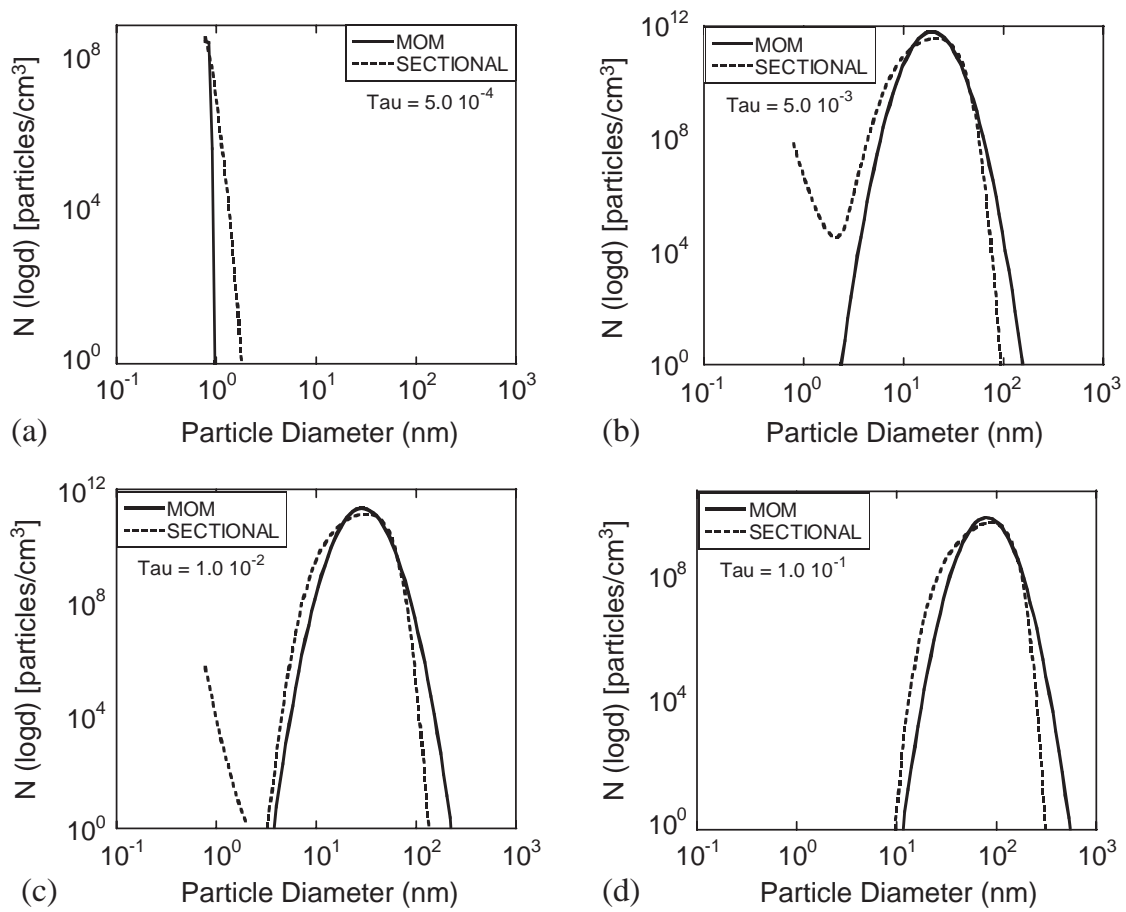

Fig. 4. Comparing particle size distributions versus particle diameter (on a logarithmic scale) from MOM and SM at different residence times in the reactor for the case shown in Fig. 3. The SM can capture the bimodal distribution at shorter residence time, while SM and MOM both approach the self-preserving size distribution at longer residence time.

noting that near the nucleation burst at $2 \mathrm{~ms}$, the silane conversion is over $60 \%$, while the particle volume fraction has reached less than $40 \%$ of its final value. Thus, at that time, more than $20 \%$ of the silicon atoms are in the form of intermediate molecules larger than silane but smaller than the assumed critical nucleus size of 10 silicon atoms. A simple mass balance on the total system confirms that final volume fraction predicted by all three methods is consistent with complete conversion of the silane to particles. After $90 \mathrm{~ms}$, when coagulation is the only active mechanism, the aerosol dynamics is decoupled from chemical kinetics. Particle diameter increases in a two-step process: rapid increase due mainly to surface growth at residence times below about $2 \mathrm{~ms}$, followed by a slower increase mainly due to coagulation, as shown in Fig. 3c. From the timescales of the various mechanisms involved, it can be observed that nucleation, growth and coagulation are occurring sequentially, but with substantial overlaps between them.

Particle size distributions at different residence times $(\tau)$ in the reactor are shown in Fig. 4. It should be noted that for the SM, particle size distribution is plotted at the mid-point of each volume bin $\left(V_{\text {mid }}\right)$, while for the MOM the size distribution is evaluated at the same volume points used for the SM plots. As mentioned earlier, QMOM does not imply a size distribution by itself and hence QMOM results are not shown in this comparison. It can be seen from Fig. 4 that at small residence time the size distributions resulting from MOM and the SM are quite different. The SM is able to capture the bimodal distribution at earlier stages when both particle nucleation and coagulation 


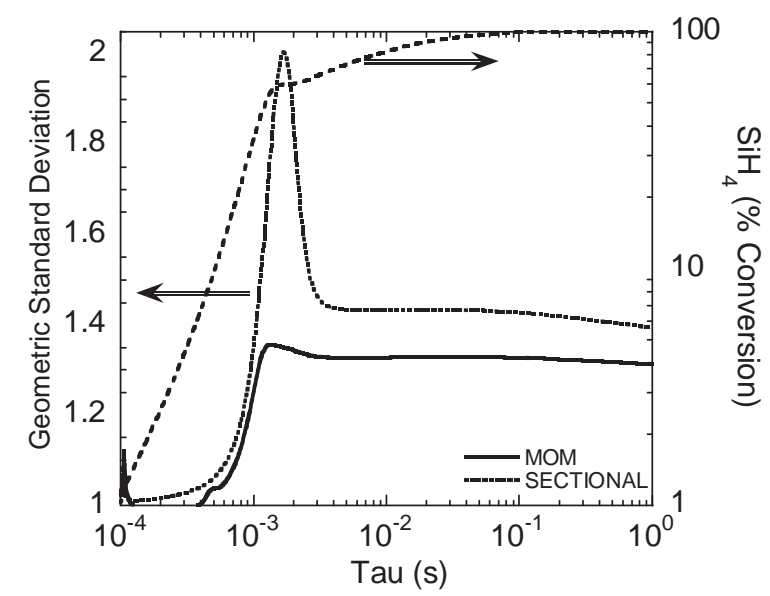

Fig. 5. Evolution of geometric standard deviation with increasing residence time for the MOM and SM. Note the sudden increase in the standard deviation around $2 \mathrm{~ms}$ (for the SM) that corresponds to a time near that shown in Fig. $4 \mathrm{~b}$ where both nucleation and coagulation modes exist.

are taking place. The smaller peak is due to the nucleation mode while the larger peak is a result of coagulation. Even though coagulation and surface growth is the dominant mechanism after $2 \mathrm{~ms}$ as mentioned earlier, some nucleation continues through about $90 \mathrm{~ms}$ when silane conversion is complete. Thus, there is some degree of overlap present between nucleation and coagulation that gives rise to this bimodal peak. Bimodal distributions at short times have also been observed in other systems (Park et al., 1999; Xiong \& Pratsinis, 1991). At longer residence time, the SM agrees with the MOM and looks almost lognormal due to the attainment of the self-preserving size distribution, which predicts at that sufficiently long times, coagulating particles reach a distribution which is independent of the initial conditions reaching the same value irrespective of the specific system considered (Friedlander, 2000). Fig. 5 shows the polydispersity resulting from both MOM and SM. The overall trends are almost the same, except for the sudden increase in the standard deviation around $2 \mathrm{~ms}$ that corresponds to a time near that shown in Fig. $4 \mathrm{~b}$ where both nucleation and coagulation modes exist. The approximation to the size distribution by the MOM tends to reduce the maximum in the polydispersity caused by the bimodal distribution, as was also observed by Xiong and Pratsinis (1991). One interesting feature in this figure is that the standard deviation in the SM does not reach the value for self-preserving size distribution in either the free molecular or continuum regime and shows a dip towards the end. For these simulation conditions, the aerosol is approaching the transition regime (Knudsen number equal to nine for the geometric mean particle diameter at $\tau=1 \mathrm{~s}$ ) where the geometric standard deviation goes through a minimum before reaching the self-preserving limit for the continuum regime.

The dependence of the SM results on bin spacing was tested using different size bins for the same volume interval. Using a larger number of bins increases the accuracy of the solution at the cost of increased computational time (Kumar \& Ramkrishna, 1996a; Landgrebe \& Pratsinis, 1990; Lister et al., 1995). We have used 50, 100 or 150 bins (equally spaced on a log scale), which correspond to spacing factors $\left(f_{\mathrm{s}}\right)$ of $1.56,1.25$ and 1.16, respectively. As can be seen from Fig. 6, decreasing $f_{\mathrm{s}}$ slightly narrows the size distribution. For $f_{\mathrm{s}}$ equal to 1.56 (corresponding to 50 size bins), the 


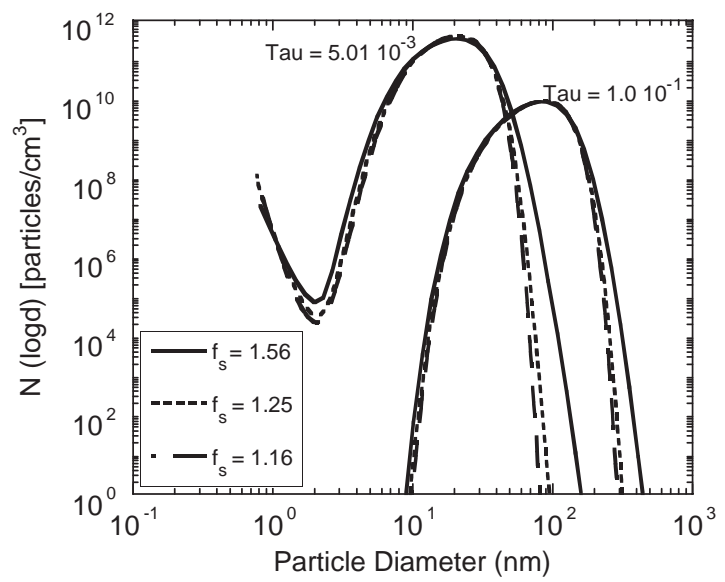

Fig. 6. Particle size distributions are plotted versus particle diameter (on a log scale) at two different residence times, for the case of simultaneous nucleation, coagulation and growth of silicon particles using the SM. Decreasing $f_{\mathrm{s}}$ increases the accuracy of the solution at the cost of increased CPU time.

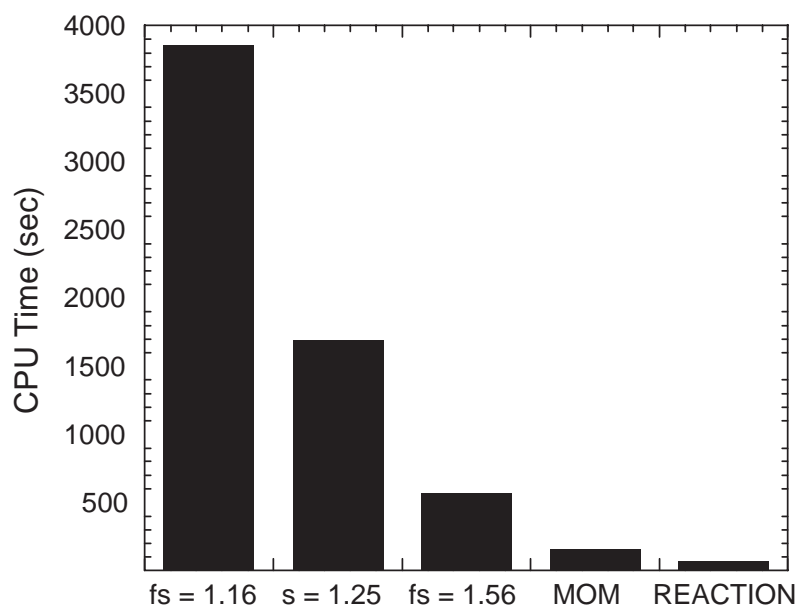

Fig. 7. Effect of gas-phase reaction and aerosol dynamics using MOM and SM (with different $f_{\mathrm{s}}$ ) on CPU time (s).

particle size distributions deviate from those with the lower $f_{\mathrm{s}}$ values, at both shorter $\left(\tau_{\mathrm{s}}=5 \mathrm{~ms}\right)$ and longer $\left(\tau_{\mathrm{s}}=100 \mathrm{~ms}\right)$ residence times. However, lowering $f_{\mathrm{s}}$ from 1.25 to 1.16 does not have much additional effect on the size distribution, though it does substantially increase the required CPU time. The very small change upon decreasing $f_{\mathrm{s}}$ from 1.25 to 1.16 indicates that the distribution is nearly converged with respect to the number of bins, which implies that numerical dispersion is not important for this problem with this bin spacing. If numerical dispersion were important, the distribution would be expected to broaden significantly with increasing spacing factor. Fig. 7 shows that decreasing $f_{\mathrm{s}}$ from 1.56 to 1.16 increases the CPU time (obtained on a SUN Blade 100 workstation, comparable in processor speed to a desktop PC) by $85 \%$ (from 564 to $3854 \mathrm{~s}$ ). 
The choice of using a reasonable number of size bins that gives accurate results without excessive computational time thus led us to select 100 bin sizes for most of our simulations. Fig. 7 also shows the effect on the CPU time of the interplay between the chemical kinetics and aerosol dynamics. If the aerosol dynamics model is omitted completely, then simulation of this complex reaction system (with 1098 reactions and 133 gas-phase species) takes $71 \mathrm{~s}$ on a SUN Blade workstation. Adding aerosol dynamics (using the MOM) to this reaction system is still computationally inexpensive (157 s), but has a bigger effect than might be expected. It adds only three additional equations to a system of over 130 equations, and yet this more than doubles the total solution time. The more complicated $\mathrm{SM}$ can take up to $3854 \mathrm{~s}$ for the same problem. Thus, unless we are interested in capturing the early stages of particle formation, MOM still remains the most viable option, especially if we are to couple aerosol dynamics with multidimensional computational fluid dynamics (CFD). Our 100 bin $\left(f_{\mathrm{s}}\right.$ equal to 1.25) SM takes about an order of magnitude more CPU time than the simple MOM approach. This further demonstrates the fact that the bottleneck to computationally feasible treatment of coupled reacting flow/aerosol dynamics problems may lie with the aerosol dynamics even when the chemical reaction mechanism is rather large and complex. The simple, reasonably accurate yet computationally efficient, sectional model proposed here can help to alleviate this bottleneck while retaining the ability to predict the details of the size distribution at short times. The size distribution at short times is particularly important when one is using short reaction times to synthesize extremely small nanoparticles. In our laboratory, we use millisecond residence times to produce silicon particles with a mean diameter as small as $5 \mathrm{~nm}$. While a direct comparison to experiment is clearly not possible at this point, we can observe from these simulations that the geometric mean particle diameter reaches $5 \mathrm{~nm}$ near a residence time of $2 \mathrm{~ms}$, which is also exactly where the geometric standard deviation peaks and the MOM is least successful. Work is in progress to extract detailed temperature and velocity distributions from a 3-D CFD model of the laser-driven six-way cross reactor used in our laboratory, and to couple aerosol dynamics models to these profiles in the particle formation zone to gain a more complete understanding of the evolution of particle size distributions with changing reactor conditions.

\section{Conclusions}

A detailed model that describes nucleation, coagulation and growth of silicon particles during thermal decomposition of silane has been developed. The model includes detailed chemical clustering of silicon hydrides containing up to ten silicon atoms. The aerosol dynamics model was solved using a conventional MOM, the QMOM and a simple SM. All three methods were in excellent agreement for the particle concentration, volume fraction and mean diameter. The evolution of the particle size distribution predicted by MOM and the SM were compared. The SM is able to capture the bimodal distribution that occurs at short times, while the MOM using a lognormal distribution fails to capture this development during the early stages of particle formation, when there is competition between nucleation and coagulation. The SM developed here treats coagulation based on conserving the mean volume of colliding particles. This is computationally less expensive and easier to implement than previous methods, as the coagulation coefficient is held constant over each interval. The use of large number of sections is thus possible to provide accurate solutions without excessive computational cost. 


\section{Acknowledgements}

This work was partially funded by the National Science Foundation (CTS-0087315) and Advanced Silicon Materials Inc. The authors would like to thank Dr. S. Tsantilis and Dr. S. E. Pratsinis of The Swiss Federal Institute of Technology (ETH), Zurich for helpful discussions on the validation of the SM. The authors are grateful to anonymous reviewer \#2 for insightful comments that led to deeper understanding of this problem and improvements in this manuscript.

\section{Appendix A}

\section{A.1. Coagulation}

To calculate coagulation coefficient in Eq. (6), based on Lee and Chen (1984) and Pratsinis (1988), a harmonic mean approximation is taken between the free molecular and continuum slip regime:

$$
\begin{aligned}
& \beta\left(v_{\text {mean }, j}, v_{\text {mean }, k}\right)=\frac{\beta_{\mathrm{CR}} \beta_{\mathrm{FM}}}{\beta_{\mathrm{CR}}+\beta_{\mathrm{FM}}}, \\
& \beta_{\mathrm{CR}}\left(v_{\text {mean }, j}, v_{\text {mean }, k}\right)=\frac{2 k_{\mathrm{B}} T}{3 \mu}\left(\frac{C(v)}{v_{\text {mean }, j}^{1 / 3}}+\frac{C(v)}{v_{\text {mean }, k}^{1 / 3}}\right), \\
& C(v)=1+\frac{1.257 \lambda}{r}, \\
& \beta_{\mathrm{FM}}\left(v_{\text {mean }, j}, v_{\text {mean }, k}\right)=\left(\frac{3}{4 \pi}\right)^{1 / 6}\left(\frac{6 k_{\mathrm{B}} T}{\rho_{\text {bulk }}}\right)^{1 / 2}\left(\frac{1}{v_{\text {mean }, j}}+\frac{1}{v_{\text {mean }, k}}\right)^{1 / 2}\left(v_{\text {mean }, j}^{1 / 3}+v_{\text {mean }, k}^{1 / 3}\right)^{2},
\end{aligned}
$$

where $k_{\mathrm{B}}$ is the Boltzman's constant, $T$ the gas temperature, $C(v)$ the slip correction, $\lambda$ the mean free path of carrier gas and $r$ the particle radius. This harmonic mean approximation captures well the transition regime and gives results for pure coagulation very close to those using the Fuchs interpolation (Fuchs, 1964).

\section{A.2. Growth}

Growth in Eq. (11) is taken as a harmonic mean approximation between the free molecular and continuum slip regime:

$$
G_{\text {effective }}=\frac{G_{\mathrm{CR}} G_{\mathrm{FM}}}{G_{\mathrm{CR}}+G_{\mathrm{FM}}}
$$

where $G_{\mathrm{CR}}$ and $G_{\mathrm{FM}}$ are defined in Eq. (11), while the linear growth rate is defined by

$$
G_{\text {linear }}=\frac{\dot{S}_{\text {bulk }} \mathrm{MW}_{\text {bulk }}}{\rho_{\text {bulk }}}
$$

$\dot{S}_{\text {bulk }}$ is the molar production rate of bulk phase silicon atoms via surface reactions, $\mathrm{MW}_{\text {bulk }}$ and $\rho_{\text {bulk }}$ are the molecular weight and density of bulk silicon, respectively. 


\section{References}

Barrett, J. C., \& Webb, N. A. (1998). A comparison of some approximate methods for solving the aerosol general dynamic equation. Journal of Aerosol Science, 29, 31.

Broadbelt, L. J., Stark, S. M., \& Klein, M. T. (1994). Computer generated reaction modeling: On-the-fly calculation of the species' properties using computational quantum chemistry. Chemical Engineering Science, 49, 4991.

Broadbelt, L. J., Stark, S. M., \& Klein, M. T. (1996). Computer generated reaction modeling: Decomposition and encoding algorithms for determining species uniqueness. Computers and Chemical Engineering, 20, 113.

Coltrin, M. E., Kee, R. J., Rupley, F. M., \& Meeks, E. (1996). SURFACE CHEMKIN-III: A FORTRAN package for analyzing heterogeneous chemical kinetics at a solid-surface-gas-phase interface. Sandia National Laboratory Report, SAND96-8217.

Diemer, R. B., \& Olson, J. H. (2002). A moment methodology for coagulation and breakage problems: Part 2-moment models and distribution reconstruction. Chemical Engineering Science, 57, 2211.

Frenklach, M., \& Harris, S. J. (1987). Aerosol dynamics modeling using the method of moments. Journal of Colloid and Interface Science, 118, 252.

Frenklach, M., Ting, L., Wang, H., \& Rabinowtiz, M. J. (1996). Silicon particle formation in pyrolysis of silane and disilane. Israel Journal of Chemistry, 36, 293.

Friedlander, S. K. (2000). Smoke, dust and haze (2nd ed.). New York: Oxford University Press.

Friedlander, S. K., \& Wang, C. S. (1966). The self-preserving particle size distribution for coagulation by Brownian motion. Journal of Colloid and Interface Science, 22, 126.

Fuchs, N. A. (1964). Mechanics of aerosols. New York: Pergamon.

Gelbard, F., \& Seinfeld, J. H. (1978). Numerical solution of the dynamic equation for particulate systems. Journal of Computational Physics, 28, 357.

Gelbard, F., \& Seinfeld, J. H. (1980). Simulation of multicomponent aerosol dynamics. Journal of Colloid and Interface Science, $78,485$.

Gelbard, F., Tambour, Y., \& Seinfeld, J. H. (1980). Sectional representations for simulating aerosol dynamics. Journal of Colloid and Interface Science, 76, 541.

Girshick, S. L., Swihart, M. T., Suh, S. M., Mahajan, M. R., \& Nijhawan, S. (2000). Numerical modeling of gas-phase nucleation and particle growth during chemical vapor deposition of silicon. Journal of the Electrochemical Society, 147, 2303.

Gordon, R. G. (1968). Error bounds in equilibrium statistical mechanics. Journal of Mathematical Physics, 9 , 655.

Ho, P., Coltrin, M. E., \& Breiland, W. G. (1994). Laser-induced flourescence measurements and kinetic analysis of Si atom formation in a rotating disk chemical vapor deposition reactor. Journal of Physical Chemistry, 98, 10138.

Hounslow, M. J., Ryall, R. L., \& Marshall, V. R. (1988). A discretized population balance for nucleation, growth, and aggregation. AIChE Journal, 34, 1821.

Hulburt, H. M., \& Katz, S. (1964). Some problems in particle technology. Chemical Engineering Science, $19,555$.

Johannessen, T., Pratsinis, S. E., \& Livbjerg, H. (2000). Computational fluid-particle dynamics for the flame synthesis of alumina particles. Chemical Engineering Science, 55, 177.

Kee, R. J., Rupley, F. M., Meeks, E., \& Miller, J. A. (1996). CHEMKIN-III: A FORTRAN chemical kinetics package for the analysis of gas-phase chemical and plasma kinetics. Sandia National Laboratory Report, SAND96-8216. $\mathrm{UC}-405$.

Kim, Y. P., \& Seinfeld, J. H. (1990). Simulation of multicomponent aerosol condensation by the moving sectional method. Journal of Colloid and Interface Science, 135, 185.

Kostoglou, M., \& Karabelas, A. J. (1994). Evaluation of zero order methods for simulating particle coagulation. Journal of Colloid and Interface Science, 163, 420.

Kremer, D. M., Davis, R. W., Moore, E. F., \& Ehrman, S. H. (2003). A numerical investigation of the effects of gas-phase particle formation on silicon film deposition from silane. Journal of Crystal Growth, 247, 333.

Kumar, S., \& Ramkrishna, D. (1996a). On the solution of population balance equations by discretization-I. A fixed pivot technique. Chemical Engineering Science, 51, 1311.

Kumar, S., \& Ramkrishna, D. (1996b). On the solution of population balance equations by discretization-II. A moving pivot technique. Chemical Engineering Science, 51, 1333. 
Kumar, S., \& Ramkrishna, D. (1997). On the solution of population balance equations by discretization-III. Nucleation, growth and aggregation of particles. Chemical Engineering Science, 52, 4659.

Landgrebe, J. D., \& Pratsinis, S. E. (1990). A discrete-sectional model for particulate production by gas-phase chemical reaction and aerosol coagulation in the free-molecular regime. Journal of Colloid and Interface Science, $1990,63$.

Lee, K. W., \& Chen, H. (1984). Coagulation rate of polydisperse particles. Aerosol Science and Technology, $3,327$.

Lee, K. W., Chen, H., \& Gieseke, J. A. (1984). Log-normally preserving size distribution for Brownian coagulation in the free-molecular regime. Aerosol Science and Technology, 3, 53.

Li, X., \& Swihart, M. T. (2001). Kinetic Monte Carlo simulation of homogeneous nucleation of hydrogenated silicon particles during silane decomposition. Electrochemical Society Proceedings Volume, PV 2001-13, Washington D.C., p. 455.

Li, X., He, Y., Talukdar, S. S., \& Swihart, M. T. (2003). Process for preparing macroscopic quantities of brightly photoluminescent silicon nanoparticles with emission spanning the visible spectrum. Langmuir, 19, 8490.

Lister, J. D., Smit, D. J., \& Hounslow, M. J. (1995). Adjustable discretized population balance for growth and aggregation. AIChE Journal, 41, 591.

Mahajan, M. R. (1999). Numerical modeling of particle nucleation, growth, and transport during atmospheric pressure chemical vapor deposition of silicon from silane. M.S. thesis, University of Minnesota.

Marchisio, D. L., Vigil, R. D., \& Fox, R. O. (2003). Quadrature method of moments for aggregation-breakage process. Journal of Colloid and Interface Science, 258, 322.

McGraw, R. (1997). Description of aerosol dynamics by the quadrature method of moments. Aerosol Science and Technology, 27, 255.

McGraw, R., Nemesure, S., \& Schwartz, S. E. (1998). Properties and evolution of aerosols with size distributions having identical moments. Journal of Aerosol Science, 29, 761.

Moody, E., \& Collins, L. R. (2003). Effect of mixing on the nucleation and growth of titania particles. Aerosol Science and Technology, 37, 403.

Muhlenweg, H., Gutsch, A., Schild, A., \& Pratsinis, S. E. (2002). Process simulation of gas-to-particle-synthesis via population balances: Investigation of three models. Chemical Engineering Science, 57, 2305.

Nijhawan, S., McMurry, P. H., Swihart, M. T., Suh, S. M., Girshick, S. L., Campbell, S. A., \& Brockmann, J. E. (2003). An experimental and numerical study of particle nucleation and growth during low-pressure thermal decomposition of silane. Journal of Aerosol Science, 34, 691.

Park, K. S., Lee, B. W., \& Choi, M. (1999). An analysis of aerosol dynamics in the modified chemical vapor deposition. Aerosol Science and Technology, 31, 258.

Petzold, L. R. (1982). A Description of DASSL: A DifferentiallAlgebraic System Solver. Sandia National Laboratory Report, SAND82-8637.

Prakash, A., Bapat, A. P., \& Zachariah, M. R. (2003). A simple numerical algorithm and software for solution of nucleation, surface growth and coagulation problems. Aerosol Science and Technology, 37, 892.

Pratsinis, S. E. (1988). Simultaneous nucleation, condensation, and coagulation in aerosol reactors. Journal of Colloid and Interface Science, 124, 416.

Pratsinis, S. E., \& Kim, K. (1989). Particle coagulation, diffusion and thermophoresis in laminar tube flows. Journal of Aerosol Science, 20, 101.

Schild, A., Gutsch, A., Muhlenweg, H., \& Pratsinis, S. E. (1999). Simulation of nanoparticle production in premixed aerosol flow reactors by interfacing fluid mechanics and particle dynamics. Journal of Nanoparticle Research, 1, 305.

Spicer, P. T., Chaoul, O., Tsantilis, S., \& Pandis, S. P. (2002). Titania formation by $\mathrm{TiCl}_{4}$ gas phase oxidation, surface growth and coagulation. Journal of Aerosol Science, 33, 17.

Swihart, M. T., \& Girshick, S. L. (1999). Thermochemistry and kinetics of silicon hydride cluster formation during thermal decomposition of silane. Journal of Physical Chemistry B, 103, 64.

Talukdar, S. S., Ng, C. A., \& Swihart, M. T. (2003). Aerosol dynamics modeling and computational fluid dynamics of a laser-driven nanoparticle synthesis reactor. Proceedings of Electrochemical Society (International Symposium of Chemical Vapor Deposition XVI and EUROCVD 14), PV 2003-08, Paris, France, p. 235.

Vemury, S., Kusters, K. A., \& Pratsinis, S. E. (1994). Time-lag for attainment of the self-preserving particle size distribution by coagulation. Journal of Colloid and Interface Science, 165, 53. 
Wong, H., Li, X., Swihart, M. T., \& Broadbelt, L. J. (2003). Encoding of polycyclic Si-containing molecules for determining species uniqueness in automated mechanism generation. Journal of Chemical Information and Computer Sciences, 43, 735 .

Wu, J. J., \& Flagan, R. C. (1988). A discrete sectional solution to the aerosol dynamic equation. Journal of Colloid and Interface Science, 123, 339.

Xiong, Y., \& Pratsinis, S. E. (1991). Gas phase production of particles in reactive turbulent flows. Journal of Aerosol Science, 22, 637.

Yuuki, A., Matsui, Y., \& Tachibana, K. (1987). A numerical study on gaseous reactions in silane pyrolysis. Japanese Journal of Applied Physics, 27, 747. 